\title{
Calculating CIs
}

Parameters of Note from MPlus output:

\begin{tabular}{|l|l|l|l|l|}
\hline Parameter & Number & Variance & B & SE \\
\hline E->SA & 32 & 0.0213873 & -.518 & .146 \\
\hline E->AE & 50 & 0.0207884 & .381 & .144 \\
\hline SA->JS & 53 & 0.00332607 & -.159 & .058 \\
\hline SA->MC & 14 & 0.0405224 & .349 & .201 \\
\hline AE->JS & 55 & 0.00276054 & .166 & .053 \\
\hline AE->JP & 65 & 0.00750982 & .221 & .087 \\
\hline HighCS ->SA & 117 & 0.0434378 & -.848 & .208 \\
\hline LowCS->SA & 118 & 0.0272023 & -.187 & .165 \\
\hline HighCS->AE & 119 & 0.0422214 & .875 & .205 \\
\hline LowCS->AE & 120 & 0.0264405 & -.113 & .163 \\
\hline $\begin{array}{l}\text { Indirect HighCS } \\
\text { SA->JS }\end{array}$ & 125 & 0.00349036 & .135 & .059 \\
\hline $\begin{array}{l}\text { Indirect LowCS } \\
\text { SA->JS }\end{array}$ & 126 & 0.000802808 & .030 & .028 \\
\hline $\begin{array}{l}\text { Indirect HighCS } \\
\text { SA->MC }\end{array}$ & 131 & 0.0681803 & -.296 & .261 \\
\hline $\begin{array}{l}\text { Indirect LowCS } \\
\text { SA->MC }\end{array}$ & 132 & 0.00636329 & -.065 & .08 \\
\hline $\begin{array}{l}\text { Indirect HighCS } \\
\text { AE->JS }\end{array}$ & 128 & 0.00327429 & .145 & .057 \\
\hline $\begin{array}{l}\text { Indirect LowCS } \\
\text { AE->JS }\end{array}$ & 129 & 0.000761701 & -.019 & .028 \\
\hline $\begin{array}{l}\text { Indirect HighCS } \\
\text { AE->JP }\end{array}$ & 134 & 0.00781479 & .193 & .088 \\
\hline $\begin{array}{l}\text { Indirect LowCS } \\
\text { AE->JP }\end{array}$ & 135 & 0.00138789 & -.025 & .037 \\
\hline
\end{tabular}


Indirect effects

1. $\quad$ E->SA->JS $(32,53)$

Coefficients:

$-.518$

$-.159$

Covariance Matrix (a, ab, b)

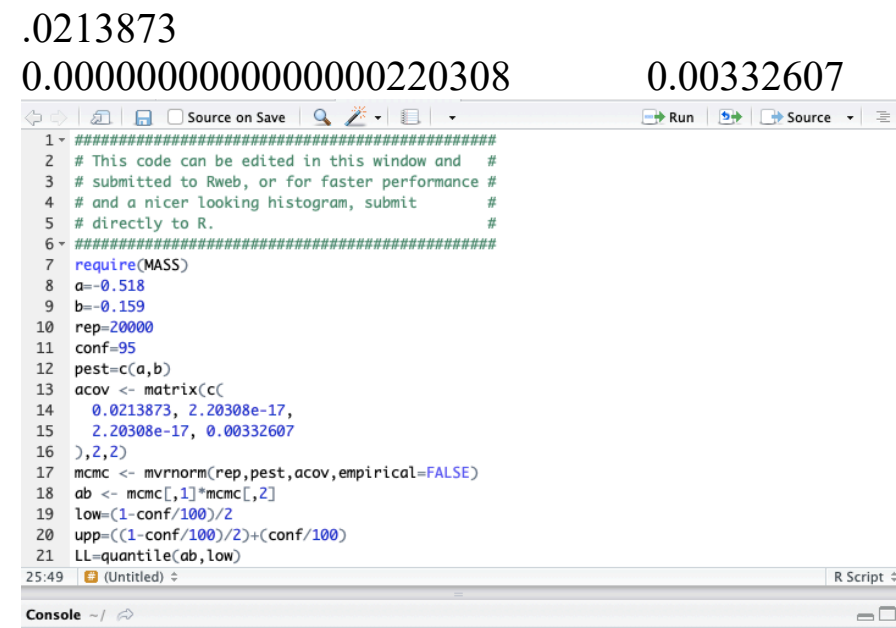

.0213873

mcmc <- mvrnorm(rep, pest, acov, empirical=FALSE)

ab <- mcmc [,1]*mcmc [, 2$]$

> upp $=((1-\operatorname{conf} / 100) / 2)+($ conf/100)

- LL=quantile(ab, low)

> UL=quantile (ab, upp)

UL4=format(UL, digits=4)

(3)

\# The number of columns in the histogram can

$\#$ an integer value.

"

> hist(ab, breaks='FD', col='skyblue ', xlab=paste(conf, '\% Confidence Interval ', 'LL', LL4,' UL' ,UL4), main='Distribution of Indirect Effect')

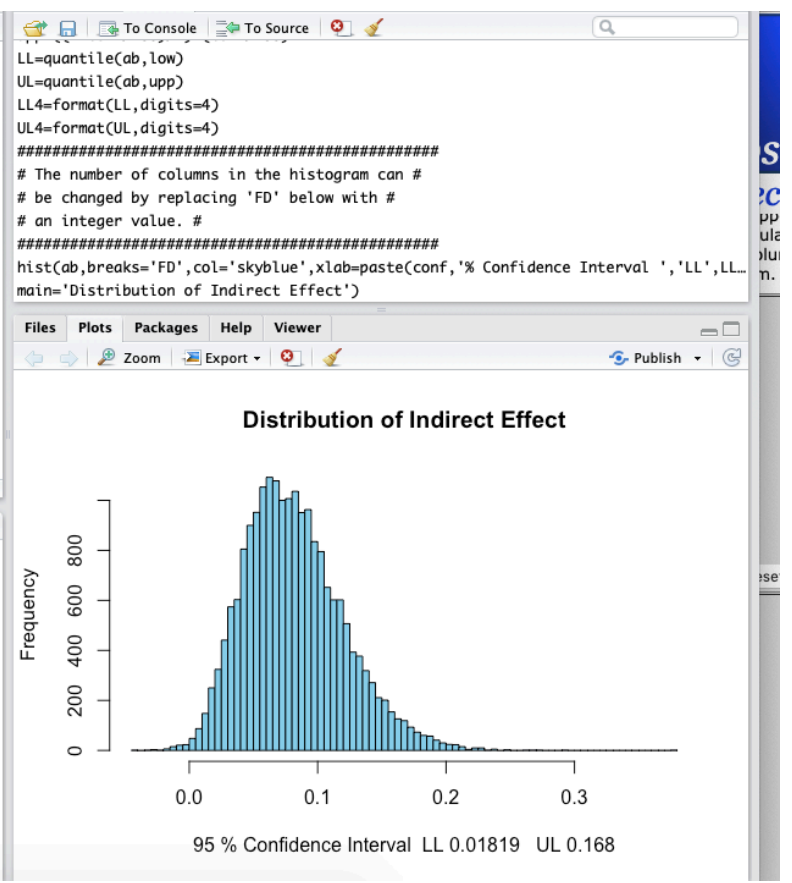

2. $\quad$ E->AE->JS $(50,55)$

Coefficients:

.381

.166

Covariance Matrix (a, ab, b)

.0207884

$-0.0000000000000000136402 \quad 0.00276054$ 


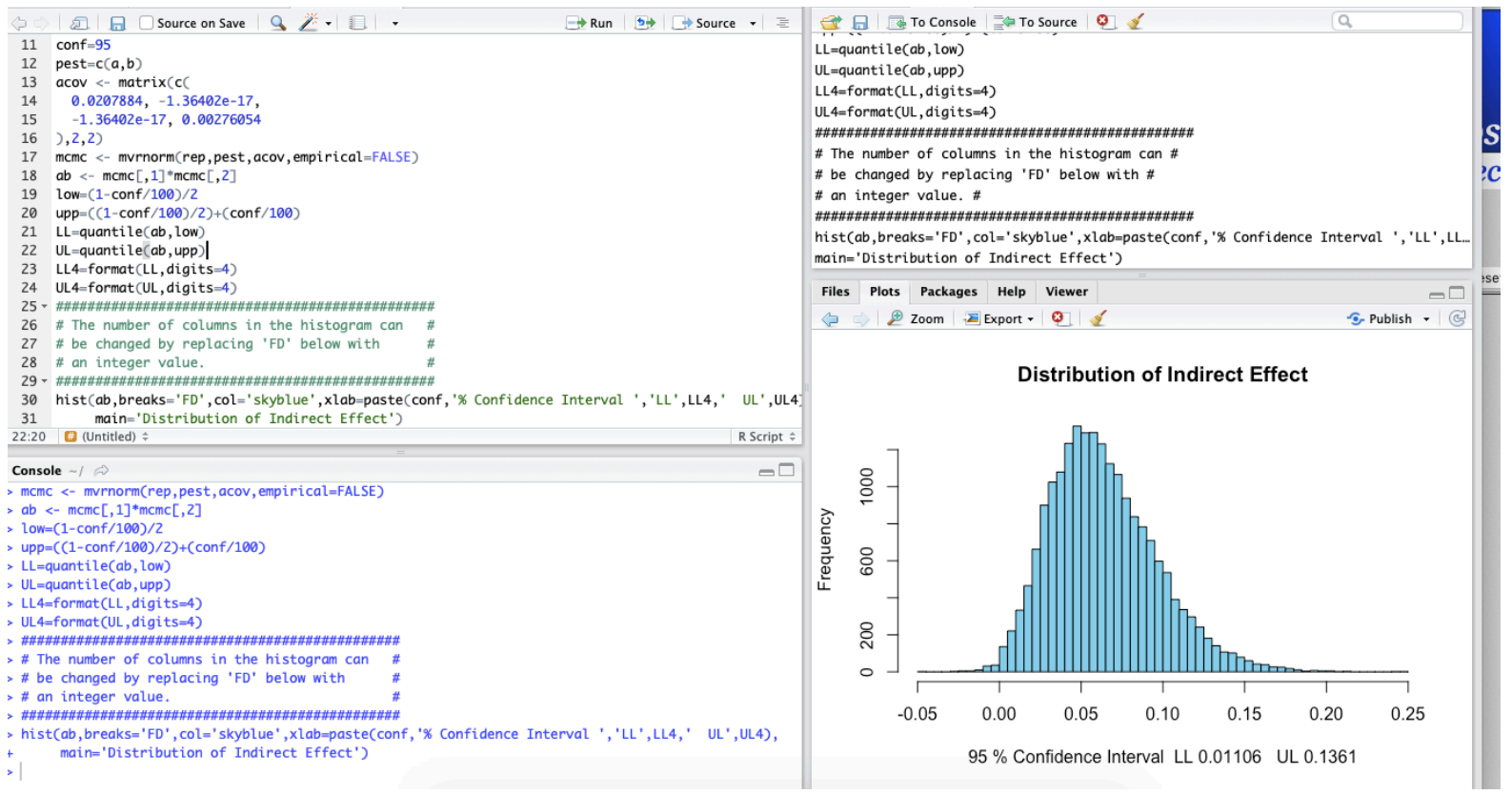

\section{3. $\quad$ E->SA->MC $(32,14)$}

Coefficients:

$-.518$

.349

Covariance Matrix (a, ab, b)

\section{.0213873}
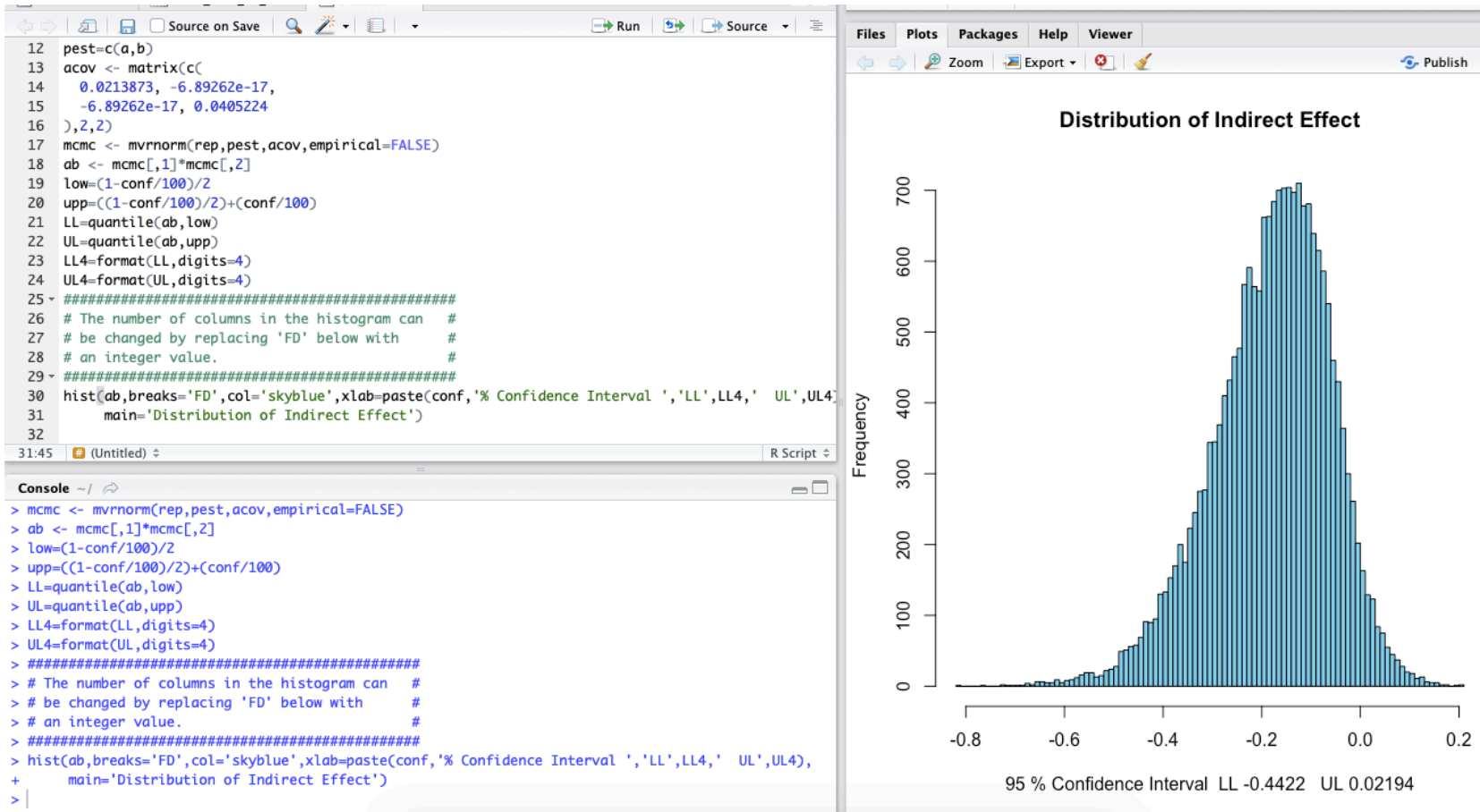
4. $\quad$ E->AE->JP $(50,65)$

Coefficients

.381

.221

Covariance Matrix

.0207884

0.0000000000000000254366

.00750982
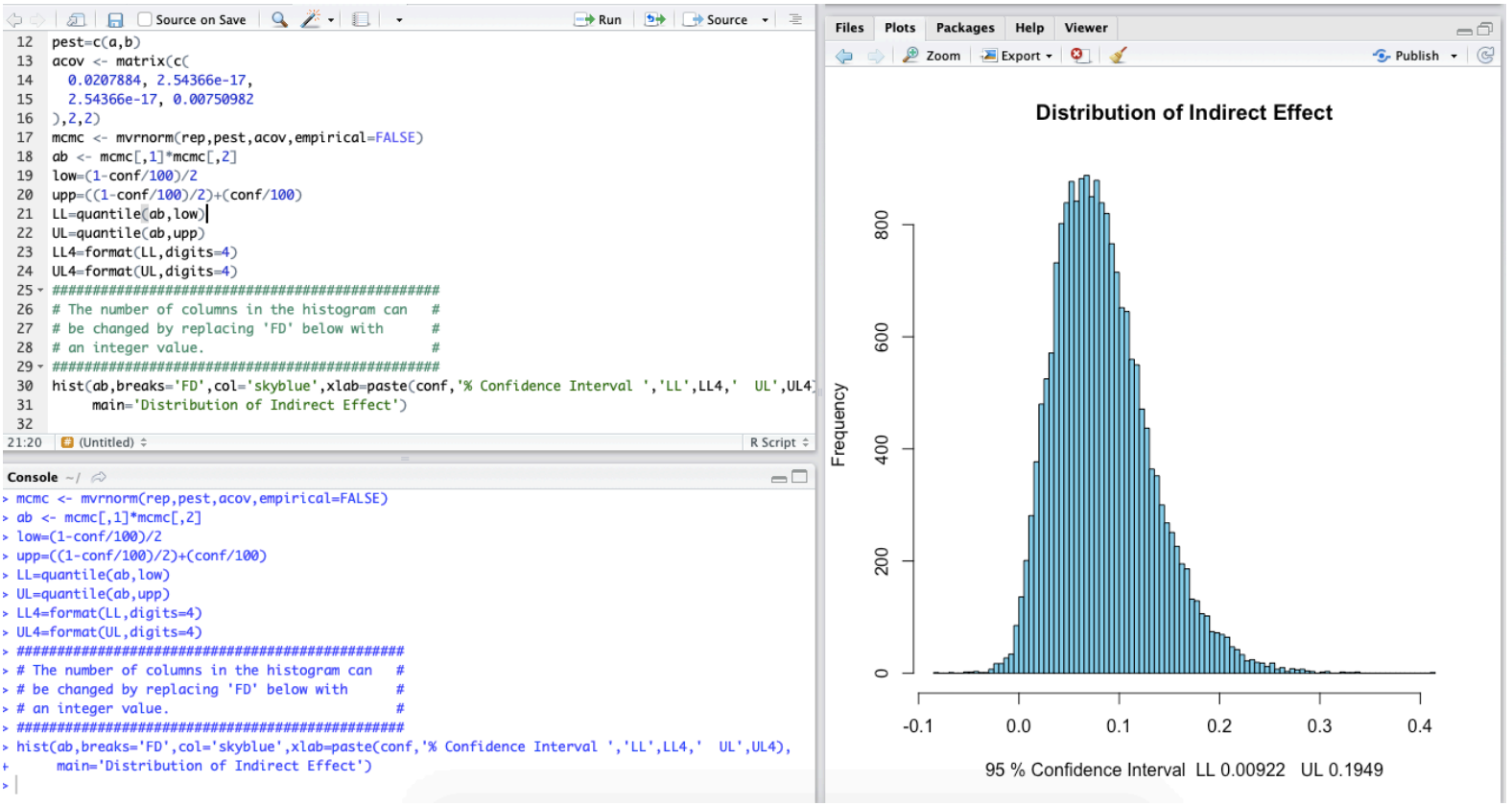

Conditional Indirect Effects \& Differences

1. High CS E- $>$ SA- $>$ JS

Coefficients $(117,53)$

$-.848$

$-.159$

Covariance Matrix (a, ab, b)

.0434378

0.0000000000000000259753

.00332607 

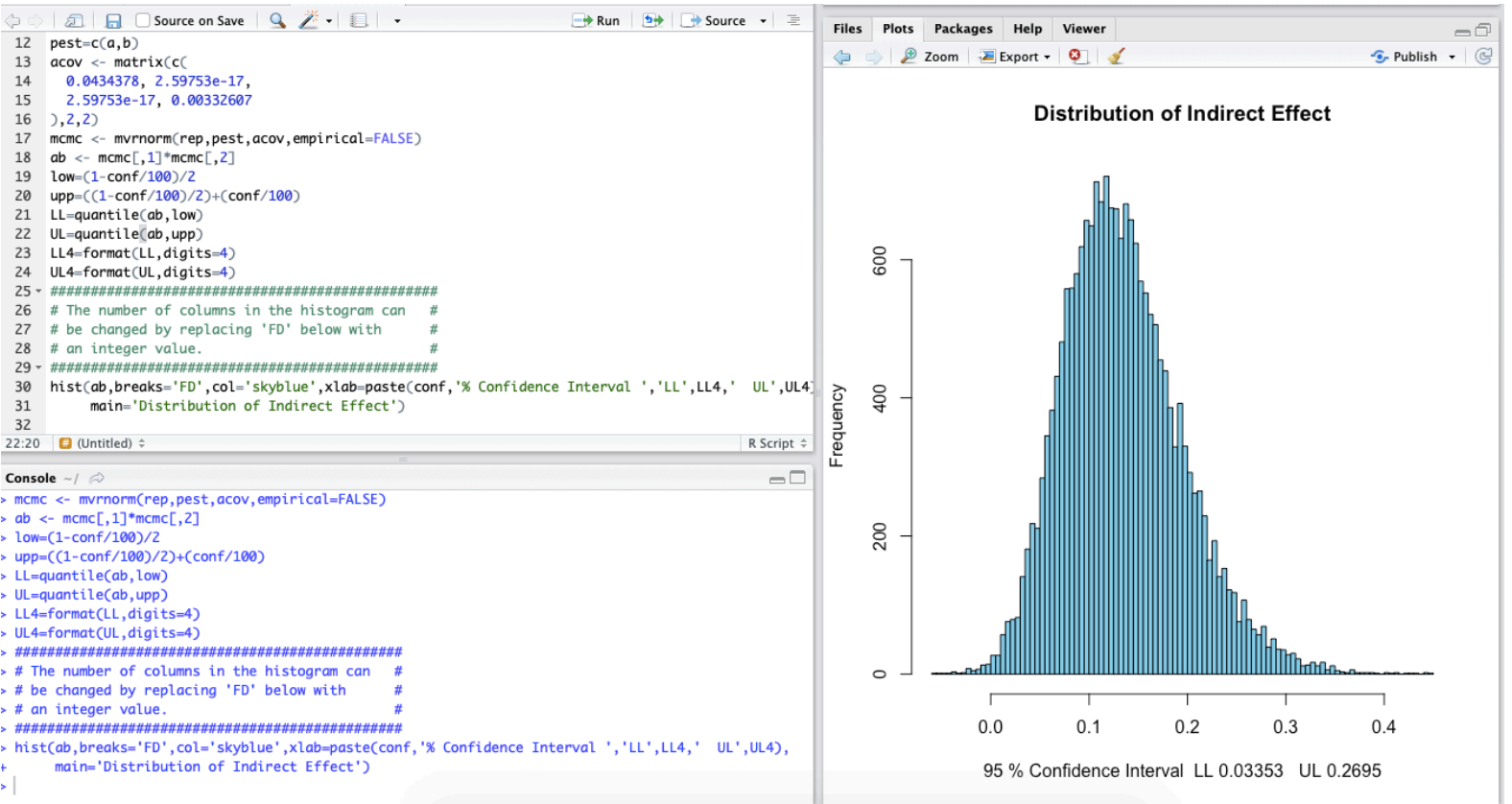

2. Low CS E->SA->JS

Coefficients $(118,53)$

$-.187$

$-.159$

\section{Covariance Matrix}

.0272023
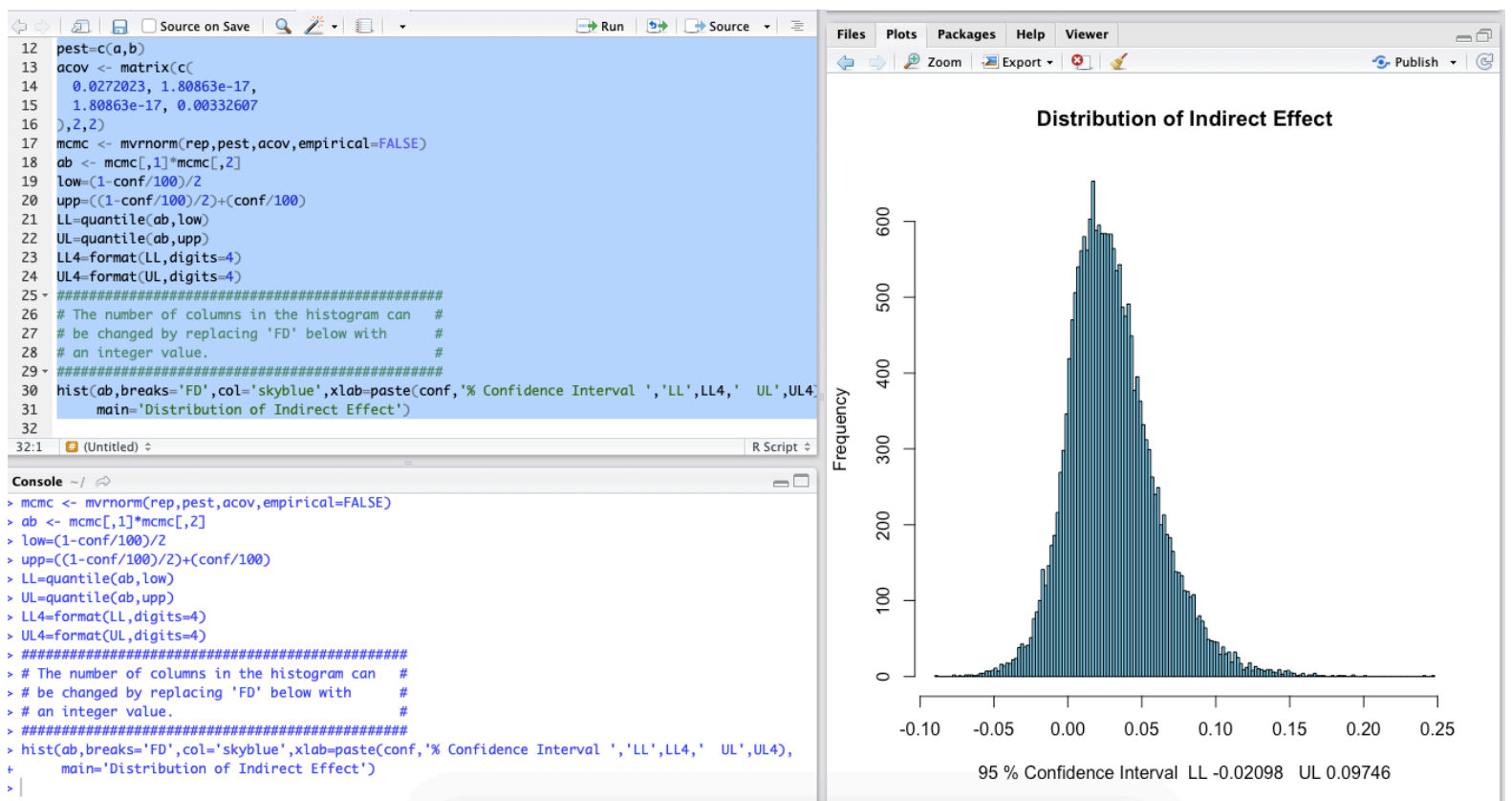

3. Difference:

Coefficients $(125,126)$ 
Covariance Matrix

0.00349036

$0.000715201 \quad 0.000802808$

$95 \%$ CI
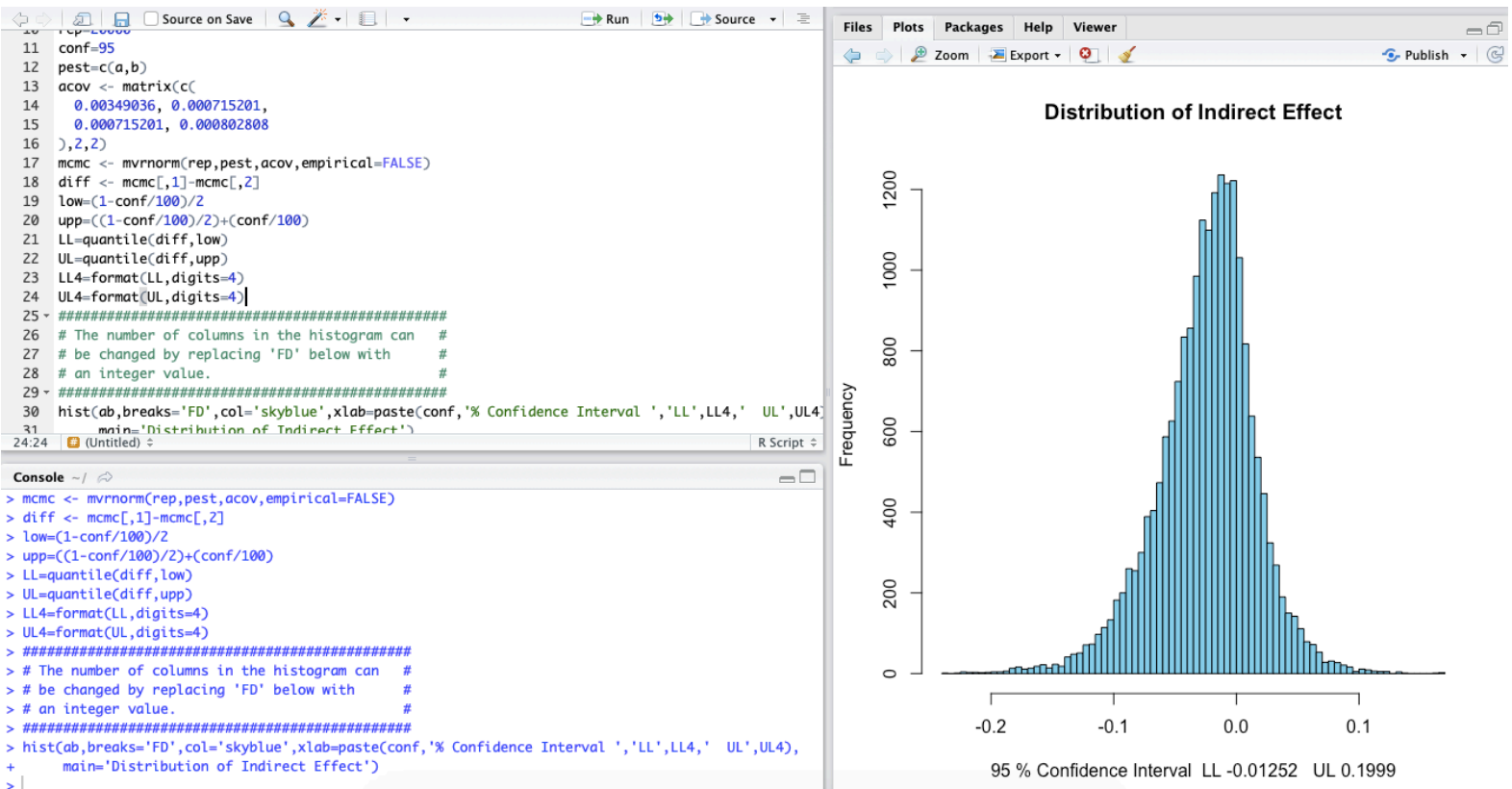

$4 \quad 0.00349036,0.000715201$

$0.000715201,0.00080280$

mcmc <- mvrnorm(rep, pest, acov, empirical=FALSE)

UL4=format (UL,

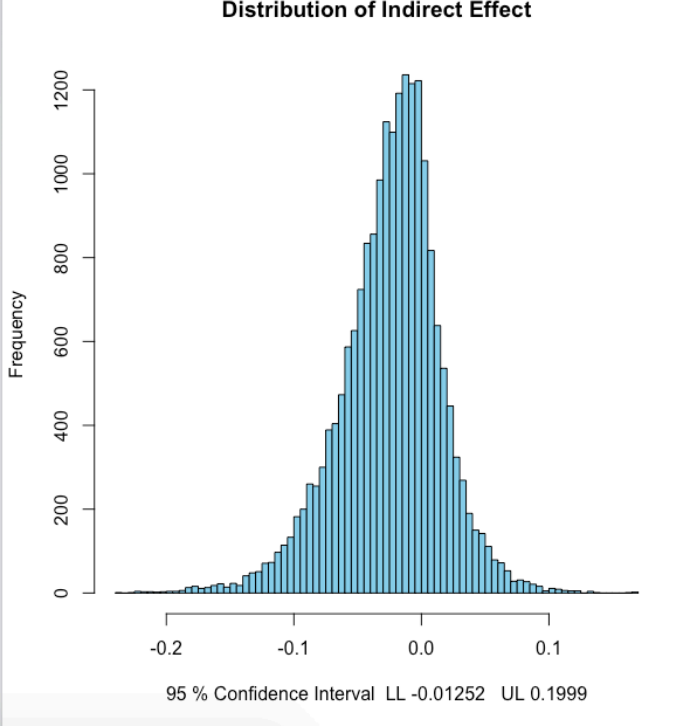

\section{$90 \% \mathrm{CI}$}

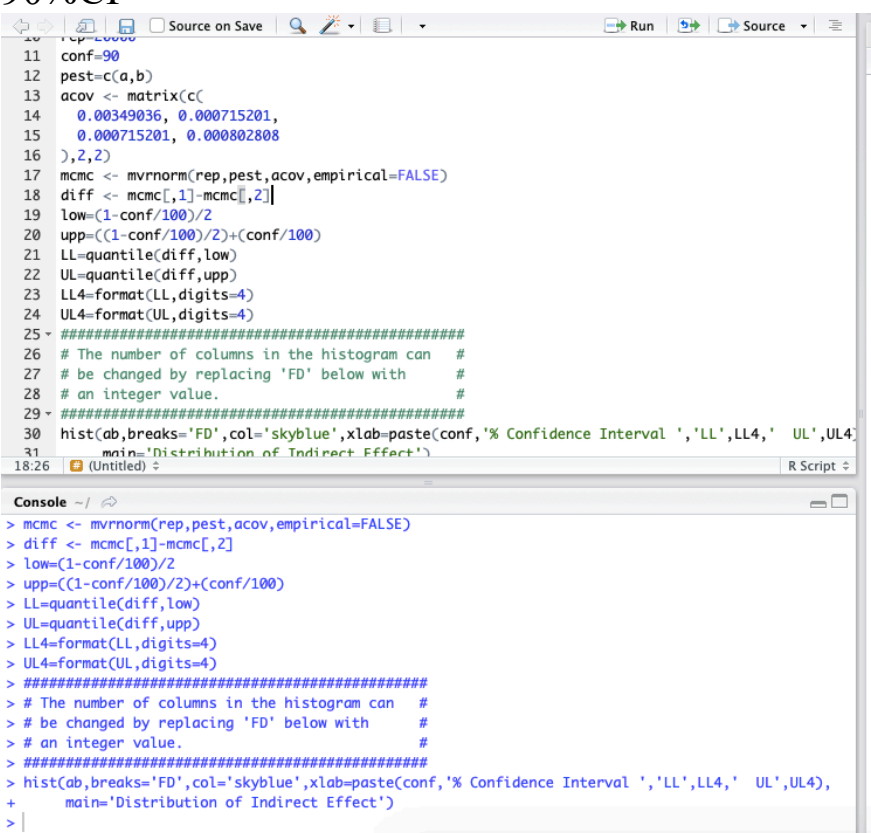

Files Plots Packages Help Viewer

a 9 Zoom $\triangle$ Export - Q

Distribution of Indirect Effect

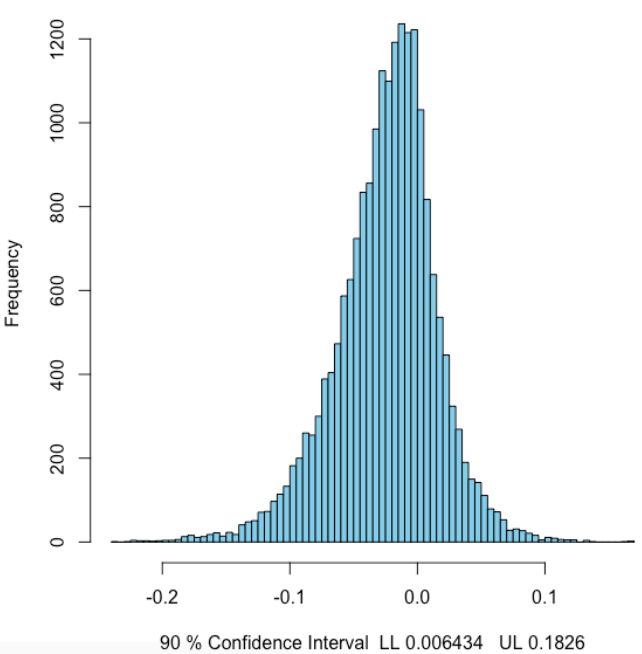

4. High CS E->AE->JS

Coefficients $(119,55)$

.875 


\section{Covariance Matrix}

\section{.0422214}

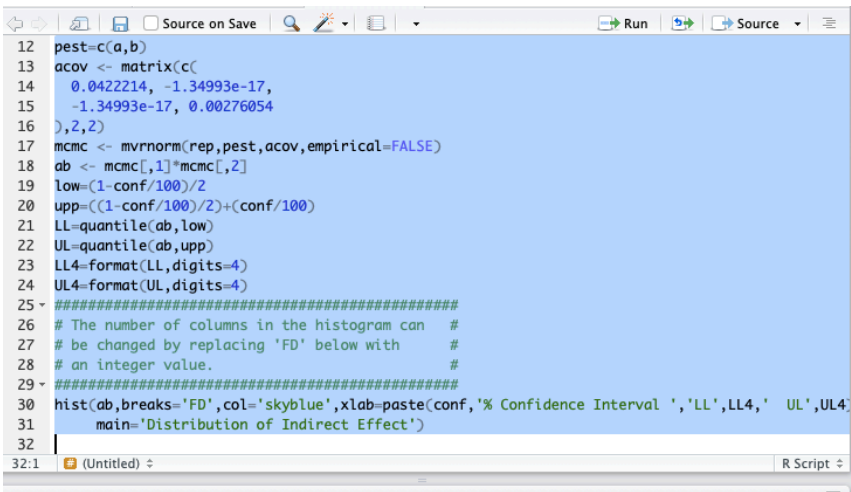

mcmc <- mvrnorm(rep, pest, acov, empirical=FALSE)

ab <- mcmc[, 1$] *$ mcmc [, 2 ,

, upp $=((1-$ conf $/ 100) / 2)+(\operatorname{conf} / 100)$

UL=quantile(ab, upp)

UL4=format (UL, digits $=4$ )

(3)

\# The number of cotumns in the histogram can

\# \# an integer value.

hist(ab, breaks='FD', col='skyblue', xlab=paste(conf, '\% Confidence Interval ', 'LL', LL4, ' UL', UL4), main='Distribution of Indirect Effect'

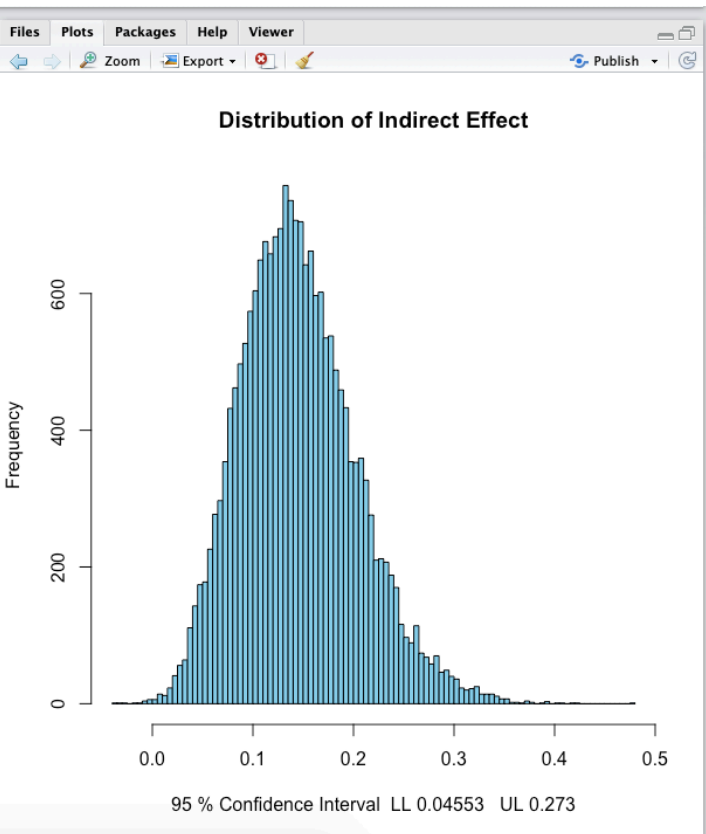

5. Low CS E->AE- $>$ JS $(120,55)$

$-.113$

.166

Covariance Matrix

.0264405

$-0.000000000000000013781 .00276054$
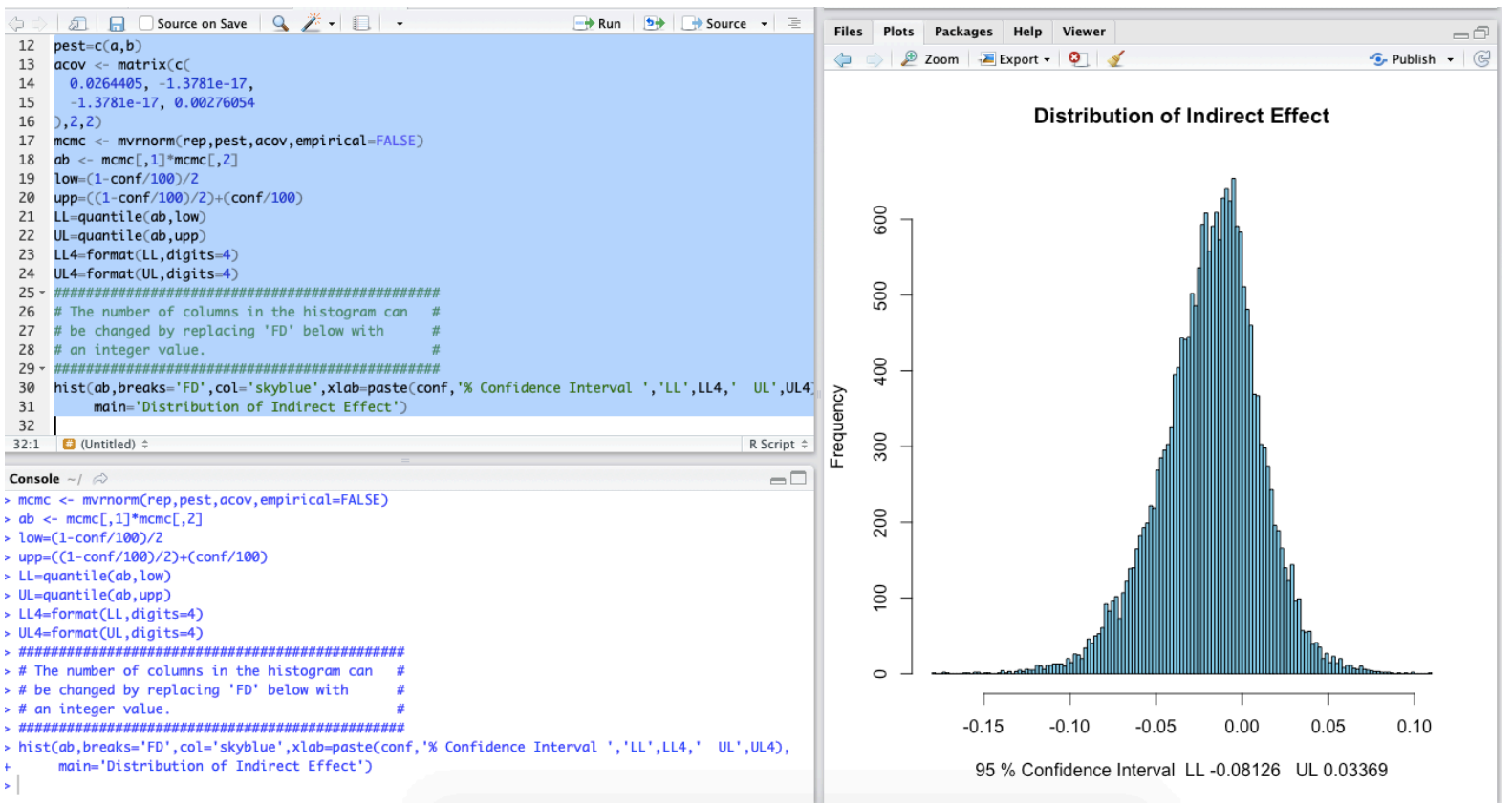
6. Difference

Coefficients (128 129)

.145

$-.019$

Covariance Matrix

0.00327429

$-0.0000733703 \quad 0.000761701$

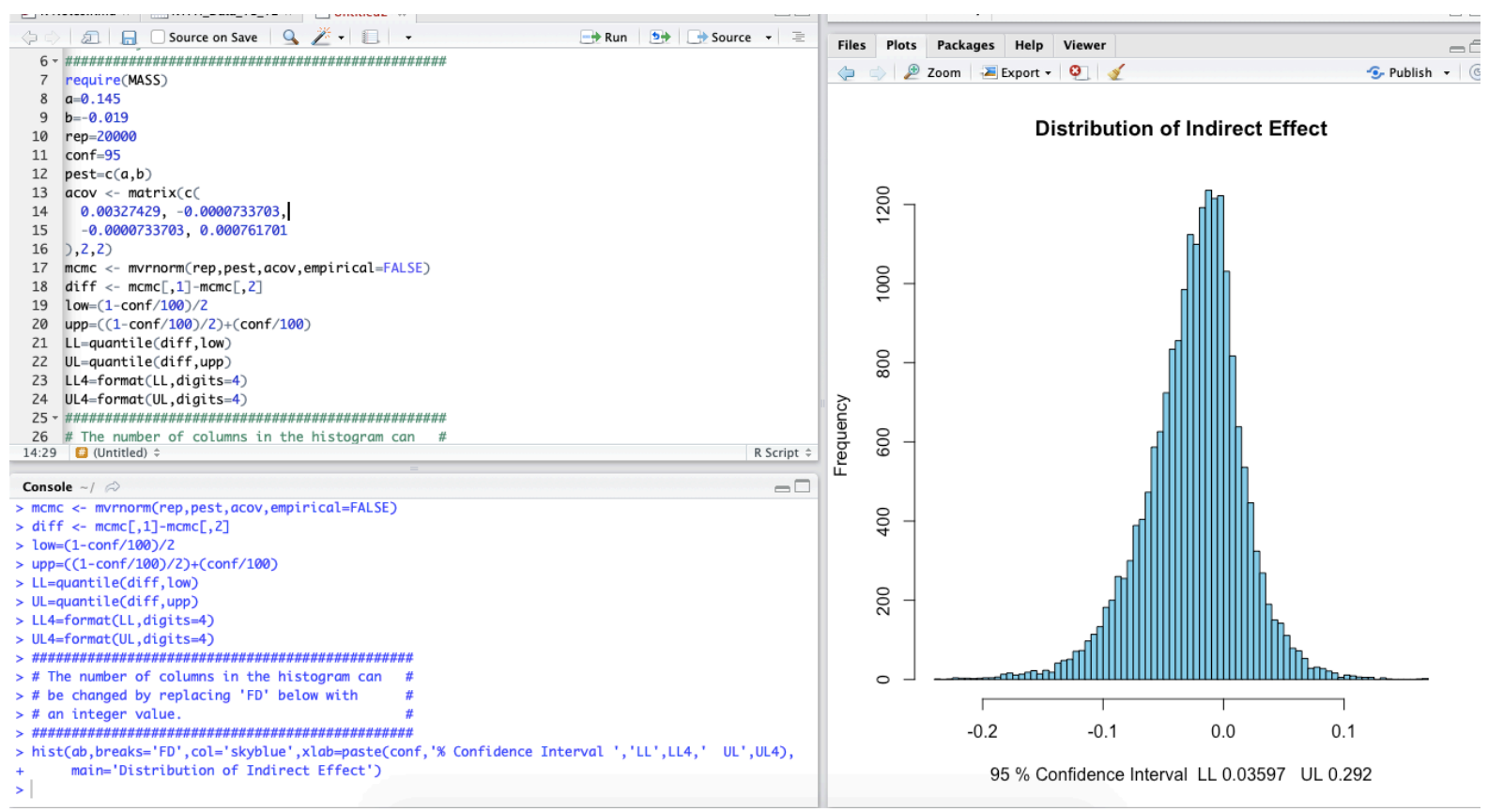

7. High CS E->SA->MC

Coefficients $(117,14)$

$-.848$

.349

Covariance Matrix

.0434378

$-0.0000000000000000779864$

.0405224 


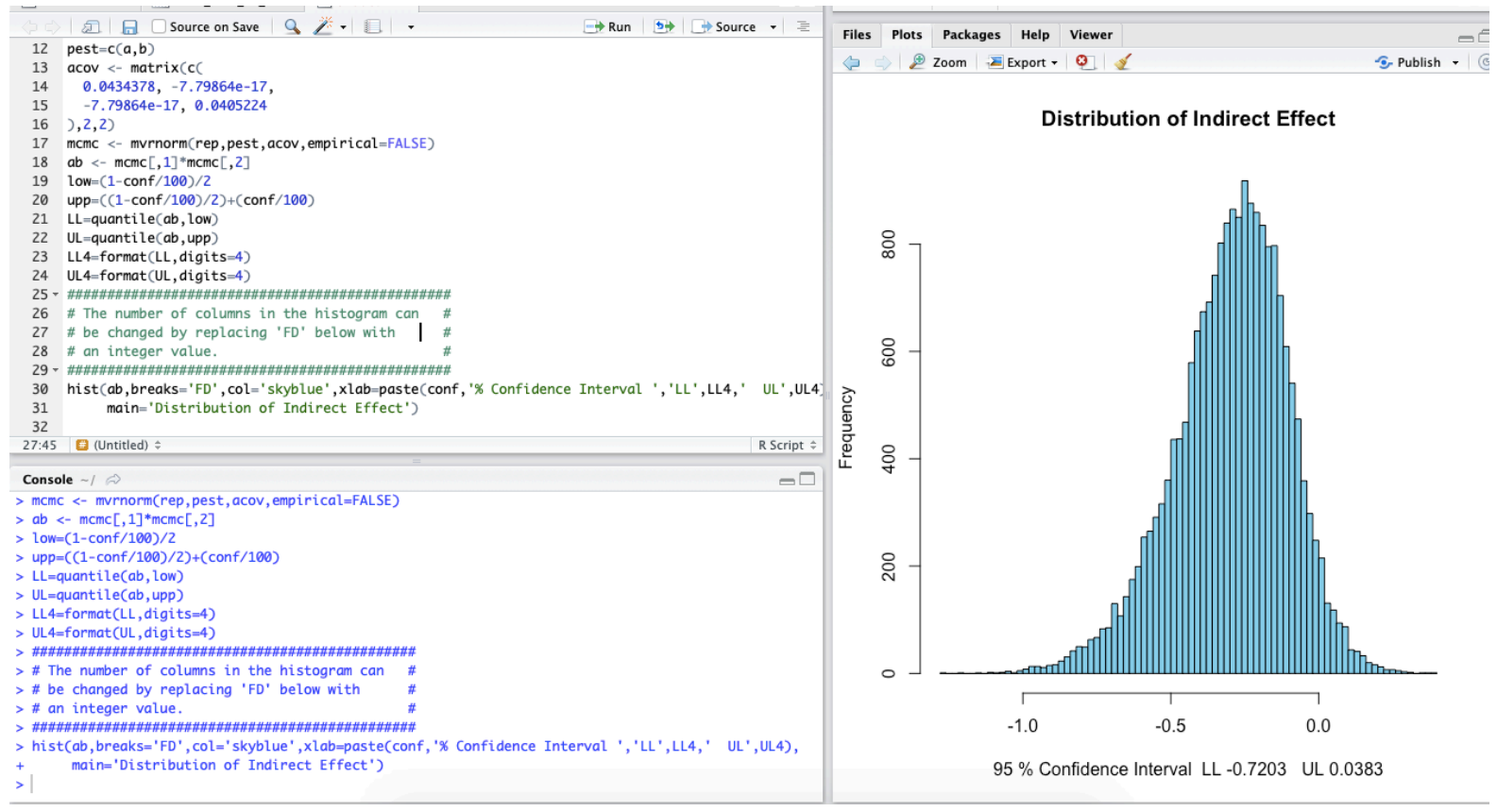

\section{Low CS E->SA MC}

Coefficients $(118,14)$

$-.187$

.349

\section{Covariance Matrix}

.0272023

$-0.0000000000000000598659$

.0405224
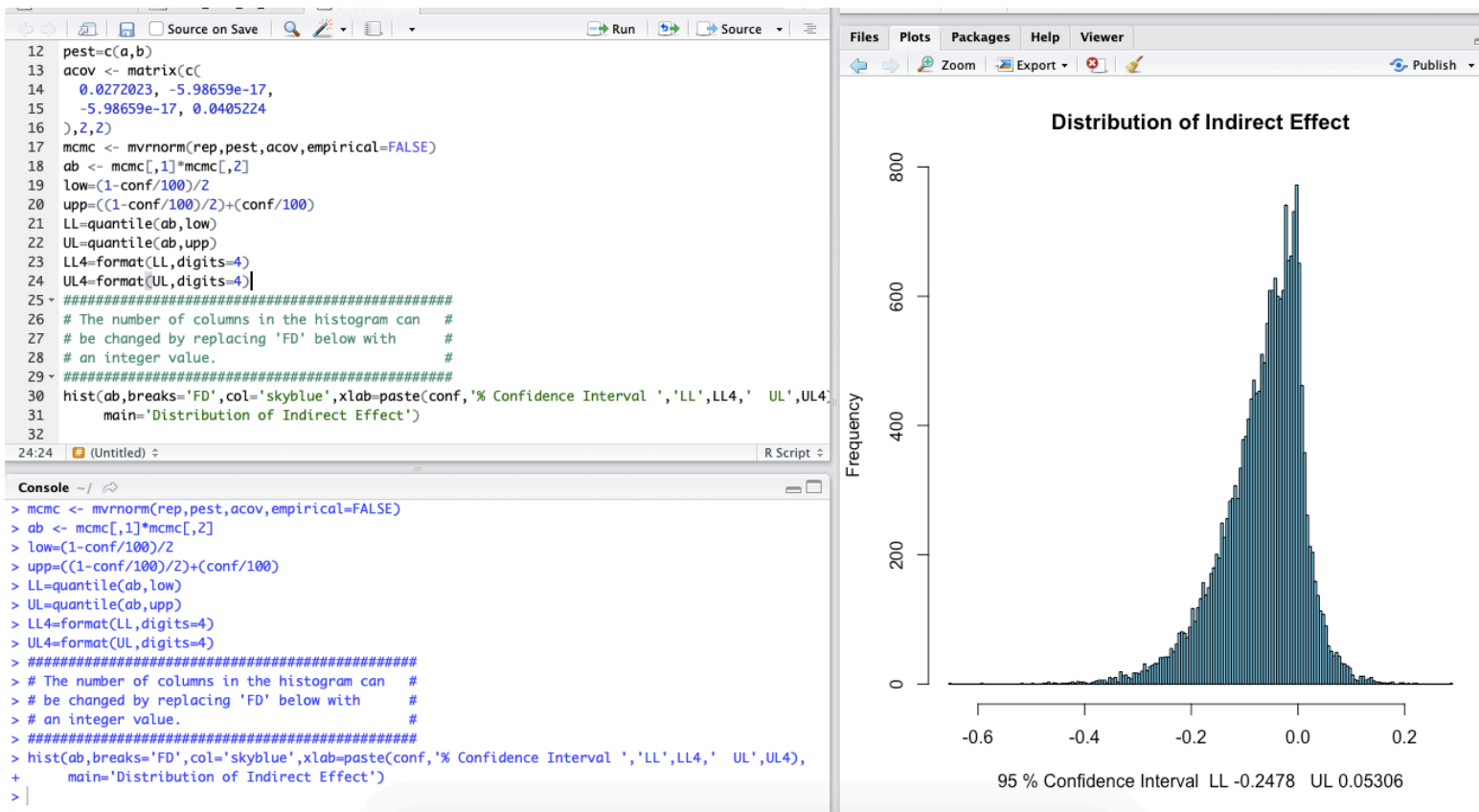

9. Difference

Coefficients $(131,132)$

$-.296$ 
Covariance Matrix

0.0681803

$\begin{array}{lll}0.0147537 & 0.00636329\end{array}$
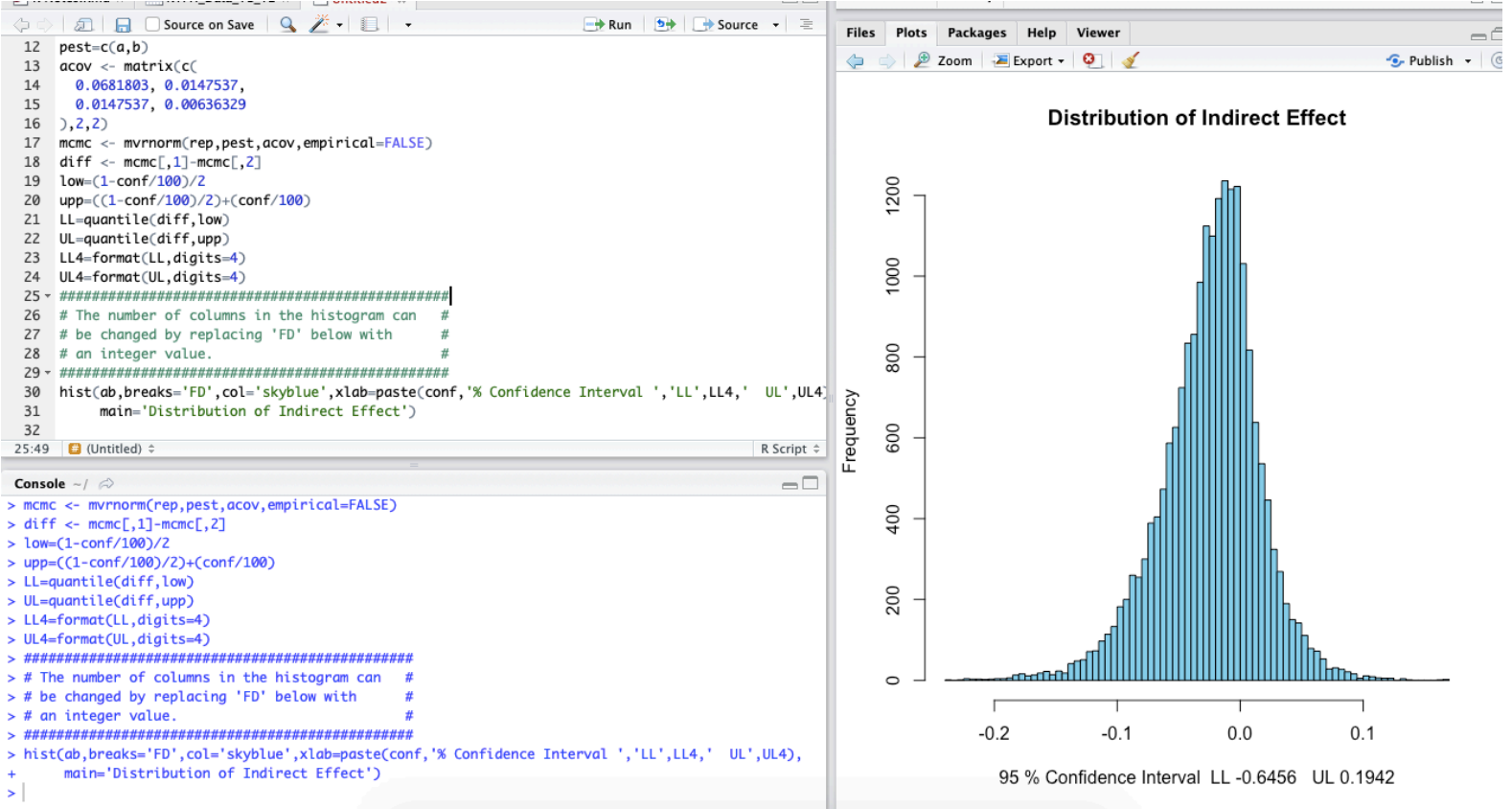

10. High CS E->AE- $>$ JP

Coefficients $(119,65)$

.875

.221

Covariance Matrix

.0422214

0.0000000000000000304381

.00750982 


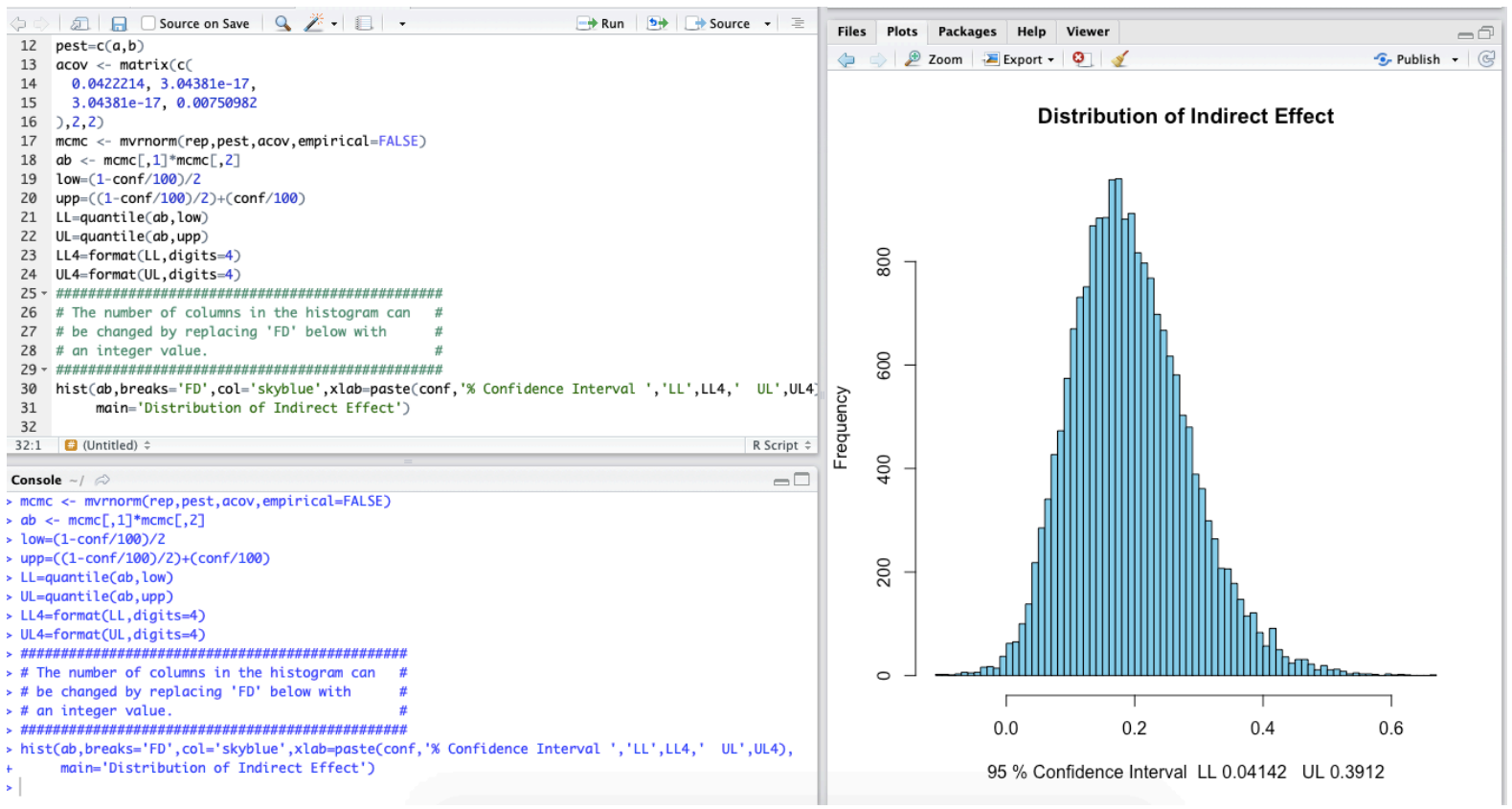

11. Low CS E->AE->JP

Coefficients $(120,65)$

$-.113$

.221

Covariance Matrix

.0264405

$0.0000000000000000204351 \quad 0.00750982$
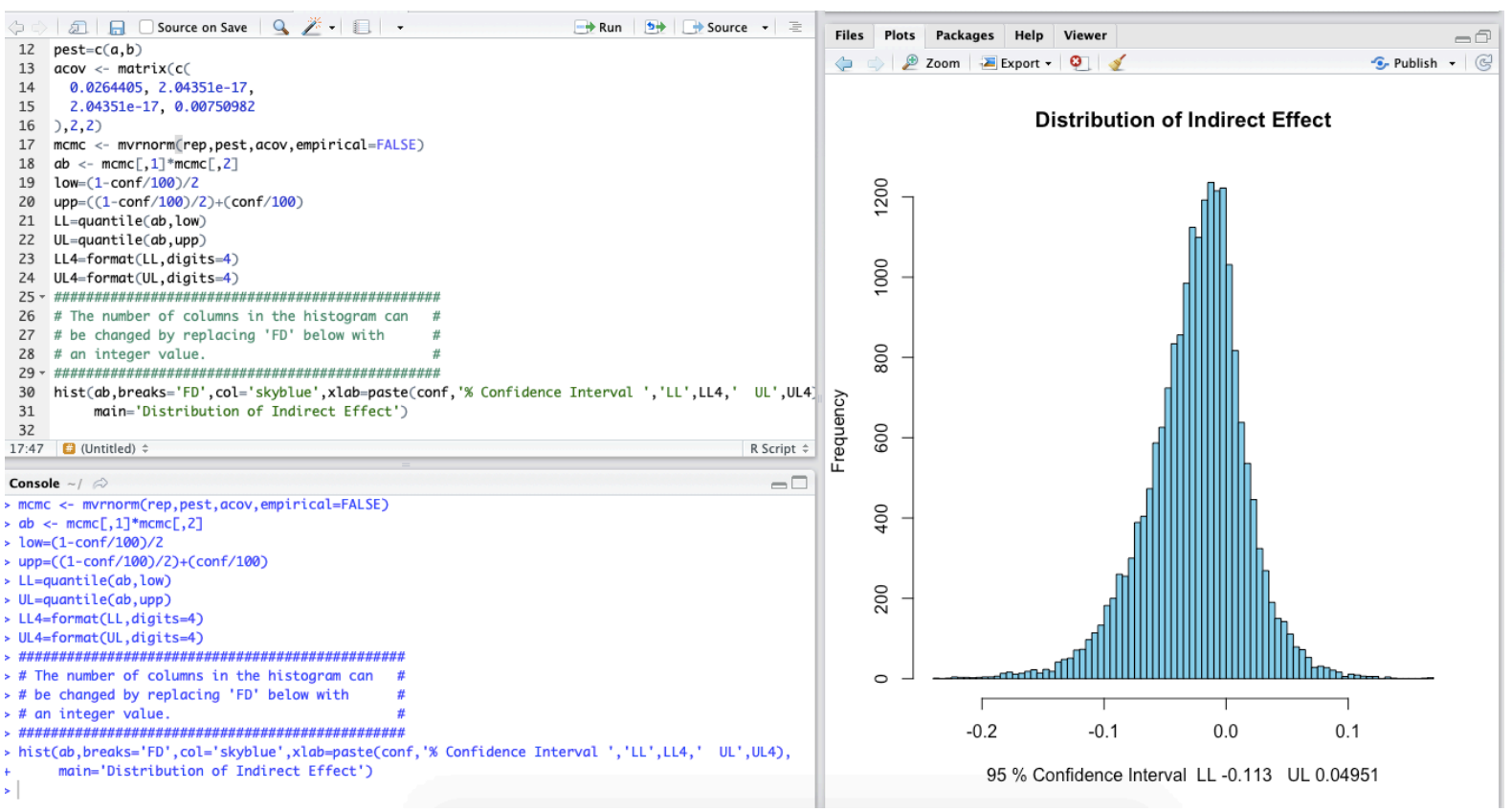

12. Difference:

Coefficients (134 135)

.193

$-.025$ 
Covariance Matrix

0.00781479

$-0.0003871120 .00138789$
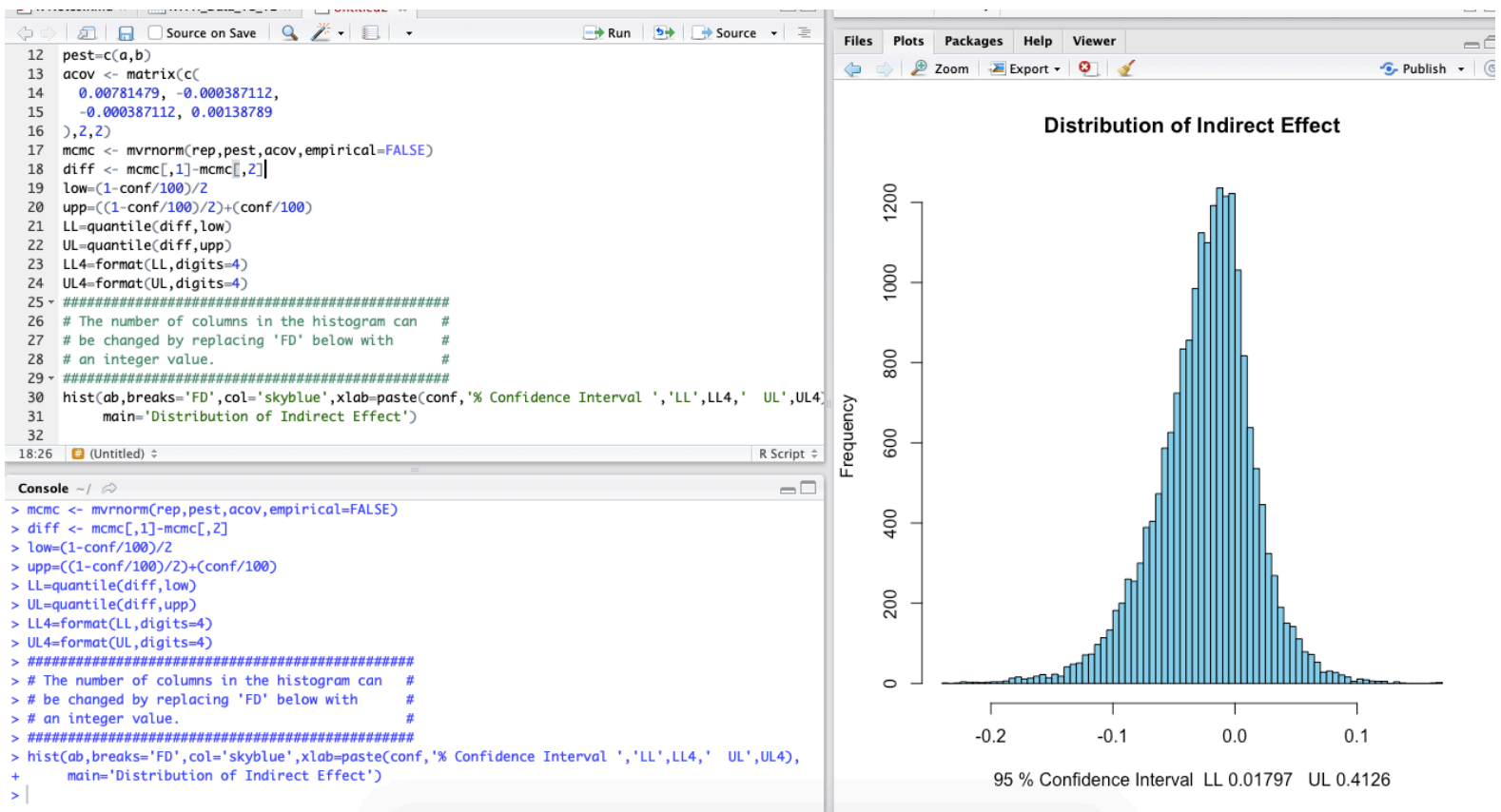